\title{
Content-Based Video Retrieval in Historical Collections of the German Broadcasting Archive
}

\author{
Markus Mühling ${ }^{1}$, Manja Meister ${ }^{4}$, Nikolaus Korfhage ${ }^{1}$, Jörg Wehling ${ }^{4}$, \\ Angelika Hörth ${ }^{4}$, Ralph Ewerth ${ }^{2,3}$, and Bernd Freisleben ${ }^{1}$ \\ 1 Department of Mathematics and Computer Science, University of Marburg, \\ Hans-Meerwein-Str. 6, D-35032 Marburg, Germany \\ \{muehling, korfhage, freisleb\}@informatik.uni-marburg.de \\ 2 German National Library of Science and Technology (TIB), \\ Welfengarten 1B, D-30167 Hannover, Germany \\ ralph.ewerth@tib.eu \\ 3 Faculty of Electrical Engineering and Computer Science, \\ Leibniz Universität Hannover, \\ Appelstr. 4, D-30167 Hannover, Germany \\ 4 German Broadcasting Archive, \\ Marlene-Dietrich-Allee 20, D-14482 Potsdam, Germany \\ \{manja.meister, joerg.wehling, angelika.hoerth\}@dra.de
}

\begin{abstract}
The German Broadcasting Archive (DRA) maintains the cultural heritage of radio and television broadcasts of the former German Democratic Republic (GDR). The uniqueness and importance of the video material stimulates a large scientific interest in the video content. In this paper, we present an automatic video analysis and retrieval system for searching in historical collections of GDR television recordings. It consists of video analysis algorithms for shot boundary detection, concept classification, person recognition, text recognition and similarity search. The performance of the system is evaluated from a technical and an archival perspective on 2,500 hours of GDR television recordings.
\end{abstract}

Keywords: German Broadcasting Archive, Automatic Content-Based Video Analysis, Content-Based Video Retrieval

\section{Introduction}

Digital video libraries become more and more important due to the new potentials in accessing, searching and browsing the data 6, 17, 2. In particular, content-based analysis and retrieval in large collections of scientific videos is an interesting field of research. Examples are Yovistd 5 . ScienceCinema ${ }^{6}$ and the TIB $\mid A V$-porta $]^{7}$ of the German National Library of Science and Technology

\footnotetext{
${ }^{5}$ http://www.yovisto.com

${ }^{6}$ http://www.osti.gov/sciencecinema

7 http://av.tib.eu
} 
(TIB). The latter provides access to scientific videos based on speech recognition, visual concept classification, and video OCR (optical character recognition). The videos of this portal stem from the fields of architecture, chemistry, computer science, mathematics, physics, and technology/engineering.

The German Broadcasting Archive (DRA) in Potsdam-Babelsberg provides access to another valuable collection of scientifically relevant videos. It encompasses significant parts of the audio-visual tradition in Germany and reflects the development of German broadcasting before 1945 as well as radio and television of the former German Democratic Republic (GDR). The DRA was founded in 1952 as a charitable foundation and joint institution of the Association of Public Broadcasting Corporations in the Federal Republic of Germany (ARD). In 1994, the former GDR's radio and broadcasting archive was established. The archive consists of the film documents of former GDR television productions from the first broadcast in 1952 until its cessation in 1991, including a total of around 100,000 broadcasts, such as contributions and recordings of the daily news program "Aktuelle Kamera", political magazines such as "Prisma" or "Der schwarze Kanal", broadcaster's own TV productions including numerous films, film adaptations and TV series productions such as "Polizeiruf 110", entertainment programs ("Ein Kessel Buntes"), children's and youth programs (fairy tales, "Elf 99") as well as advice and sports programs. Access to the archive is granted to scientific, educational and cultural institutions, to public service broadcasting companies and, to a limited extent, to commercial organizations and private persons. The video footage is often used in film and multimedia productions. Furthermore, there is a considerable international research interest in GDR and German-German history. Due to the uniqueness and importance of the video collection, the DRA is the starting point for many scientific studies.

To facilitate searching in videos, the DRA aims at digitizing and indexing the entire video collection. Due to the time-consuming task of manual video labeling, human annotations focus on larger video sequences and contexts. Furthermore, finding similar images in large multimedia archives is manually infeasible.

In this paper, an automatic video analysis and retrieval system for searching in historical collections of GDR television recordings is presented. It consists of novel algorithms for visual concept classification, similarity search, person and text recognition to complement human annotations and to support users in finding relevant video shots. In contrast to manual annotations, content-based video analysis algorithms provide a more fine-grained analysis, typically based on video shots. A GDR specific lexicon of 91 concepts including, for example, "applause", "optical industry", "Trabant", "military parade", "GDR emblem" or "community policeman" is used for automatic annotation. An extension of deep convolutional neural networks (CNN) for multi-label concept classification, a comparison of a Bag-of-Visual Words (BoVW) approach with CNNs in the field of concept classification and a novel, fast similarity search approach are presented. The results of automatically annotating 2,500 hours of GDR television recordings are evaluated from a technical and an archival perspective. 


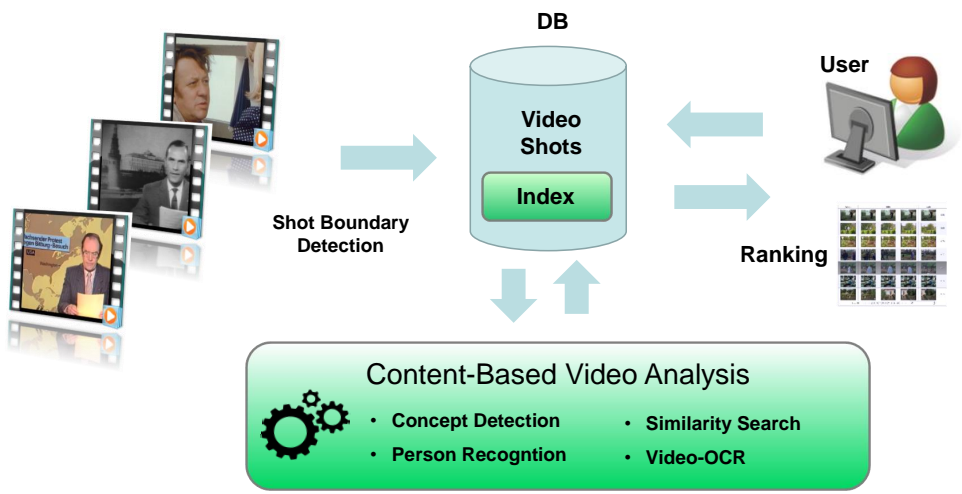

Fig. 1: Video retrieval system.

The paper is organized as follows: Section 2 describes the video retrieval system and the video analysis algorithms. In Section 3 , experimental results on the GDR television recordings are presented. Section 4 concludes the paper and outlines areas for future research.

\section{Content-Based Video Retrieval}

The aim of content-based video retrieval is to automatically assign semantic tags to video shots for the purpose of facilitating content-based search and navigation. Figure 1 shows an overview of our developed video retrieval system. First, the videos are preprocessed. This step mainly consists of shot boundary detection [9] in conjunction with thumb generation for the keyframes, which are required for later visualization purposes. The aim of shot boundary detection is the temporal segmentation of a video sequence into its fundamental units, the shots. Based on video segmentation, the following automatic content-based video analysis algorithms are applied: concept classification, similarity search, person and text recognition. The resulting metadata are written to a database and serve as an intermediate description to bridge the "semantic gap" between the data representation and the human interpretation. Given the semantic index, arbitrary search queries can be processed efficiently, and the query result is returned as a list of video shots ranked according to the probability that the desired content is present. In the following, the content based video analysis algorithms are described in more detail.

\subsection{Visual Concept Classification}

The classification of visual concepts is a challenging task due to the large complexity and variability of their appearance. Visual concepts can be, for example, objects, sites, scenes, personalities, events, or activities. The definition of the 
GDR specific concept lexicon is based on the analysis of user search queries with a focus on queries that were experienced as difficult and time-consuming to answer manually. A lexicon of 91 concepts was defined after analyzing more than 36,000 user queries received within a five-year period from 2008 to 2013. Recent user queries, assumed to be of future research interest, were summarized thematically and ordered by frequency. The concept lexicon comprises events such as "border control" and "concert", scenes such as "railroad station" and "optical industry", objects like "Trabant" or activities such as "applauding". Manually annotated training data was used to build the concept models. For this purpose, a client-server based annotation tool was built to facilitate the process of training data acquisition and to select a sufficient quantity of representative training examples for each concept. We defined a minimum number of 100 positive samples per concept.

Recently, deep learning algorithms fostered a renaissance of artificial neural networks, enabled by the massive parallel processing power of modern graphics cards. Deep learning approaches, especially deep CNNs, facilitated breakthroughs in many computer vision fields [14, 11, 4, 23]. Instead of using handcrafted features such as SIFT descriptors 16, CNNs learn the features automatically during the training process. A CNN consists of several alternating convolution and max-pooling layers with increasingly complex feature representations and typically has several fully connected final layers.

State-of-the-art network architectures for image recognition [14, 22, as well as the current datasets [8, 27] consider only a single concept per image ("singlelabel"). In contrast, real world concept classification scenarios are multi-label problems. Several concepts, such as "summer", "playground" and "teenager", may occur simultaneously in an image or scene. While some approaches use special ranking loss layers [10, we have extended the CNN architecture using a sigmoid layer instead of the softmax layer and a cross entropy loss function.

Furthermore, millions of training images are needed to build a deep CNN model from scratch. Due to the relatively small amount of available training data, we have adapted a pre-trained CNN classification model (GoogleNet 22 trained on ImageNet 8]) to the new GDR concept lexicon using our multi-label CNN extension and performed a fine-tuning on the GDR television recordings. The models were trained and fine-tuned using the deep learning framework Caffe [12. In addition, a comparison between a state-of-the-art BoVW approach and our deep multi-label CNN was performed on the publicly available, fully annotated NUSWIDE scene dataset [7]. The BoVW approach is based on a combination of different feature representations relying on optimized SIFT variants [19]. The different feature representations are combined in a support vector machine (SVM)-based classifier using multiple kernel learning [20]. Our deep multi-label CNN approach significantly outperformed the BoVW approach by a relative performance improvement of almost $20 \%$. In contrast to binary SVM classifiers, deep neural networks are inherently multi-class capable so that only a single compact model has to be built for all concept classes. While the runtime of the BoVW approach takes already 1.97 seconds for feature extraction and the classification 


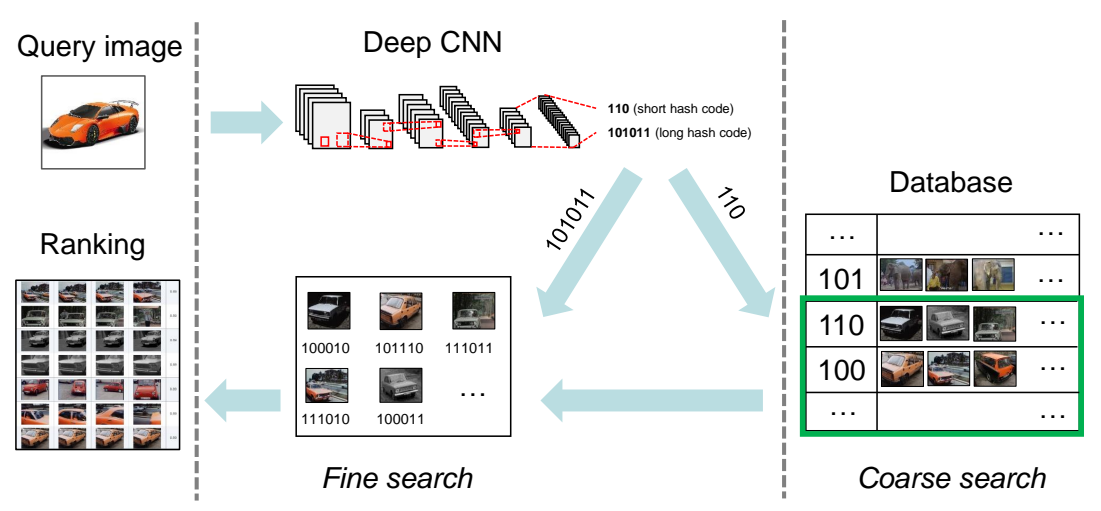

Fig. 2: Content based similarity search.

runtime depends linearly on the number of concepts, our deep multi-label CNN takes only 0.35 seconds on the CPU (Intel Core i5) and just 0.078 seconds on the GPU (Nvidia Titan X). Although deep neural networks are computationally expensive in the training phase, they are very efficient at the classification stage. Altogether, deep multi-label CNNs provide better recognition quality, compact models and faster classification runtimes.

\subsection{Similarity Search}

Since the DRA offers researchers a large number of video recordings containing several millions of video shots, the need for a system that helps to rapidly find desired video shots emerges. While scanning through the whole video archive is practically infeasible for humans, a possible solution is to index the videos via concepts as described in Section 2.1. However, this approach requires manually annotated training images for learning the concept models. Additionally, search queries are restricted to the vocabulary of predefined concepts, and new concept models have to be developed on demand. In contrast to textual conceptbased queries, image-based queries provide users more flexibility and new ways of searching.

While query-by-content based on low-level features turned out to be insufficient to search successfully in large-scale multimedia databases, image representations learned by deep neural networks greatly improved the performance of content-based image retrieval systems [25]. They are less dependent on pixel intensities and are clearly better suited for searching semantic content. However, high-dimensional CNN features are not well suited for searching efficiently in large video collections. Fast search in large databases is an essential requirement for practical use. For this purpose, proposals for learning binary image codes for compact representations and fast matching of images have been made. Krizhevsky and Hinton [13, for example, used very deep autoencoders, and Lin et al. 15] extended a CNN to learn binary hash codes for fast image retrieval. 
In this section, an approach for fast content-based similarity search in large video databases is presented. For efficient storage and matching of images, binary codes are learned by deep CNNs. This mapping of images to binary codes is often referred to as "semantic hashing" 21. The idea is to learn a "semantic hash function" that maps similar images to similar binary codes. To learn the hash function, a method similar to the approach described by Lin et al. 15] is used. Based on a pre-trained CNN classification model, we devised a coding layer and an appropriate loss layer for error propagation for the network architecture. The best results were obtained using Chatfield et al.'s network [5] trained on the PLACES dataset [27]. An advantage of using pre-trained classification models is the speed-up in training time. To obtain high-level features, we built the coding layer on top of the last fully-connected layer. Furthermore, the hash function can be adapted to unlabeled datasets by using the predictions of the pre-trained classification model for error propagation.

The overall video retrieval system is based on the analysis of keyframes, i.e., representative images. In our approach, five frames (the first, the last, and three in between) per video shot are used as keyframes for indexing. Given the hash function, the keyframes of the video collection are fed into the deep CNN and the mapped binary codes are stored in the database. Based on the resulting index, queries-by-image can be answered by matching the binary code of the given image to the database. The overall retrieval process is shown in Figure 2, Given the query image, the binary codes are extracted using the learned deep CNN. We use a two-stage approach based on semantic hashing. First, a coarse search is performed using 64-bit binary codes resulting in a "short" list of potential results. The hamming distance is applied to compare the binary codes, and a vantage point tree [26] is used as an additional index structure to accelerate the search process. The resulting short list consists of the 10,000 nearest neighbors. The longer the binary codes, the more accurate the image representations are. Therefore, in the second stage, a refined search using 256-bit binary codes is performed on the short list, and the images are ranked according to the distance to the query image. Differently from Lin et al. [15], hash codes are used for the refined search as well. In our two-stage approach, both coding layers, for 64-bit and 256-bit binary codes, are integrated into the same architecture resulting in a concurrent computation at training and testing time. Finally, the resulting images are mapped to video shots.

\subsection{Person Recognition}

Based on the analysis of user search queries, the GDR specific concept lexicon has been extended by 9 personalities, including "Erich Honecker", "Walter Ulbricht", "Hilde Benjamin", "Siegmund Jähn", "Hermann Henselmann", "Christa Wolf", "Werner Tübke", "Stephan Hermlin" and "Fritz Cremer". Instead of using concept classification, personalities are handled using a face recognition approach. Therefore, feature representations of known persons are stored in a face database, and a face recognition system was built that scans the video shots and recognizes the identity of a detected face image by comparing it to the face database. 


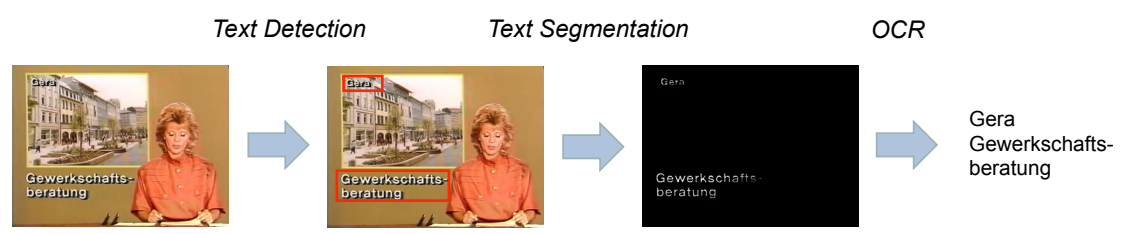

Fig. 3: Text recognition pipeline.

Finally, the resulting index of person occurrences can be used in search queries. The face processing pipeline consists of several components: face detection, face alignment and face recognition. For the face recognition component, we evaluated the following approaches: Fisherfaces [3], Local Binary Pattern Histograms [1] and a commercial library, called FaceVAC $\$^{8}$. Furthermore, we evaluated whether a preprocessing step using grayscale histogram equalization, training data augmentation using Google search queries, or a face tracking component improve the recognition accuracy. Based on the results of our evaluations, we finally used the method of Viola and Jones 24] for face detection and FaceVACs for face alignment and recognition.

\subsection{Text Recognition (Video OCR)}

Superimposed text often hints at the content of a video shot. In news videos, for example, the text is closely related to the current report, and in silent movies it is used to complement the screen action with cross headings. Involved algorithms can be distinguished by their objective, whether it is text detection, also called localization, text segmentation, or optical character recognition (see Figure 3).

We developed a text recognition system that allows users to search for inscene and overlaid text within the video archive. For this purpose, the I-frames of the videos are analyzed and the recognized ASCII text is stored in the database. Based on the resulting index, OCR search queries can be answered by a list of video shots ranked according to the similarity to the query term. Due to poor technical video quality and low contrast of text appearances, the similarities between the query term and the words in the database are calculated using the Levenshtein distance.

For text detection, localization and segmentation in video frames, a method based on Maximally Stable Extremal Regions (MSER) [18] is used. It is able to detect both overlaid text, as well as text within the scene, for example on banners. Experimental results revealed that the text segmentation component plays an important role in the case of poor quality videos. Text segmentation crops localized text out of the image to yield black letters on a white background. This step is necessary to feed the result into an OCR algorithm that transforms the

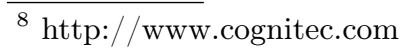




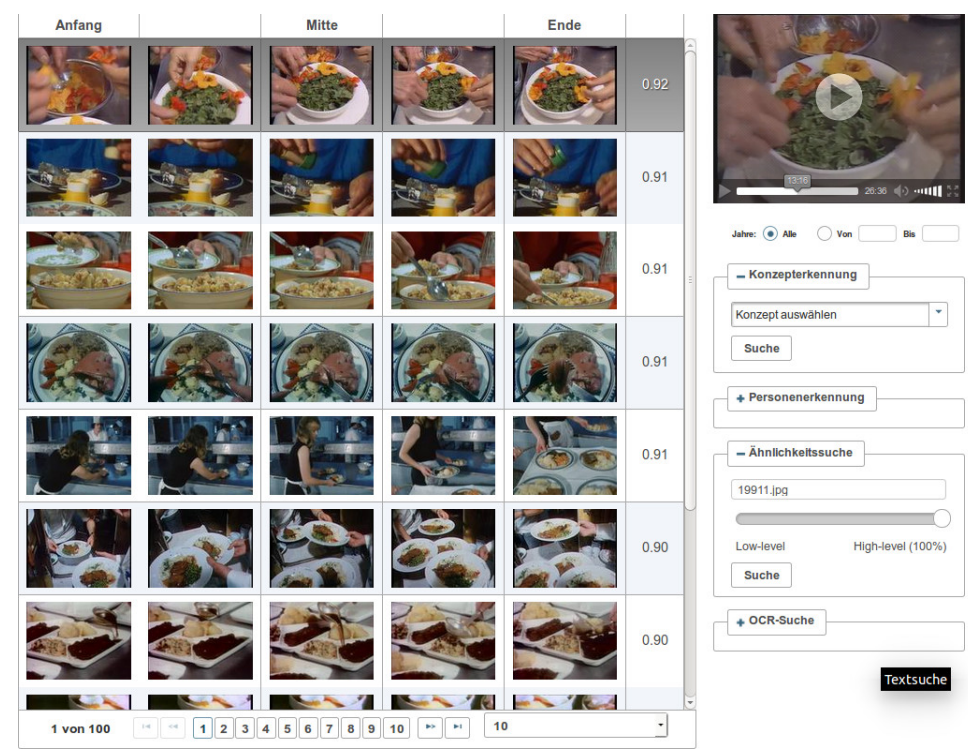

Fig. 4: Retrieval results for a query image showing a meal.

image into machine-readable text. A non-uniform background would normally impair this process. For OCR, we finally used the open source library Tesseract?

\section{Experimental Results}

A web-based GUI has been developed to automatically respond to user queries related to concepts, persons, similar images and text. The retrieval results are presented to the user in the form of a ranked list of video shots (see Figure 4) where each video shot is represented by five video frames and a probability score indicating the relevance. Furthermore, a video player allows to visually inspect the video shots.

\subsection{Data}

In total, more than 3,000 hours of historical GDR television recordings have been digitized by now. The video footage is technically very challenging. Many recordings are in grayscale and of low technical quality; the older the recordings, the poorer the video quality. The temporal segmentation of the videos resulted in approximately 2 million video shots. From these shots, 416,249 have been used for the training process and 1,545,600 video shots, corresponding to about 2,500 hours of video data, for testing.

\footnotetext{
${ }^{9}$ http://code.google.com/p/tesseract-ocr/
} 


\subsection{Results}

In the following, the results for concept classification, person recognition, similarity search and video OCR are presented. The results are evaluated using the average precision (AP) score that is the most commonly used quality measure in video retrieval. The AP score is calculated from the list of video shots as follows:

$$
A P(\rho)=\frac{1}{\left|R \cap \rho^{N}\right|} \sum_{k=1}^{N} \frac{\left|R \cap \rho^{k}\right|}{k} \psi\left(i_{k}\right) \quad \text { with } \quad \psi\left(i_{k}\right)= \begin{cases}1 & \text { if } i_{k} \in R \\ 0 & \text { otherwise }\end{cases}
$$

where $N$ is the length of the ranked shot list, $\rho^{k}=\left\{i_{1}, i_{2}, \ldots, i_{k}\right\}$ is the ranked shot list up to rank $k, R$ is the set of relevant documents, $\left|R \cap \rho^{k}\right|$ is the number of relevant video shots in the top- $k$ of $\rho$ and $\psi\left(i_{k}\right)$ is the relevance function. Generally speaking, AP is the average of the precisions at each relevant video shot. To evaluate the overall performance, the mean AP score is calculated by taking the mean value of the AP scores from different queries.

Concept Classification and Person Recognition. In total, 86 concepts, comprising 77 concepts and 9 persons, were evaluated. Of the originally 91 concepts 14 were dismissed due to an insufficient number of training images. However, another 14 of the 77 evaluated concepts have less than 100 training images. Altogether, 118,020 positive training examples were gathered for learning the concept models. The retrieval results for concepts and persons were evaluated based on the top-100 and top-200 ranked video shots. Although 14 concepts have less than 100 training images and despite poor video quality, we obtained a mean AP of $62.4 \%$ and $58.0 \%$, respectively. Even concepts occurring predominantly in grayscale shots of low video quality yielded good results, such as "daylight mining" with $84.3 \%$ AP. These results reveal the high robustness of the proposed multi-label deep CNN approach with respect to low quality historical video data.

For person recognition, we achieved a very good result of $83.3 \%$ mean AP on the top-100 and $81.1 \%$ mean AP on the top-200 video shots and even $100 \%$ AP for distinctive and frequently occurring personalities, such as Erich Honecker and Walter Ulbricht. In summary, we achieved a mean AP of $64.6 \%$ and $60.5 \%$ on the top-100 and top-200, respectively, for both concepts and persons.

Similarity Search. The interpretation whether two images are similar is subjective and context specific. The definition of similarity ranges from pixel-based similarity to image similarity based on the semantic content. How much low-level and semantic similarity contribute to the retrieval results can be individually adjusted in the GUI. Furthermore, two use cases have been implemented: searching by video frames selected from within the corpus and searching by external images, e.g., downloaded from the Internet. In our evaluation, we focus on the more difficult task of semantic similarity using 50 external query images from the Internet chosen collaboratively by computer scientists and archivists. An example result for a query image showing a meal is presented in Figure 4. Each 
retrieval result was evaluated up to the first 100 video shots obtaining a mean $\mathrm{AP}$ of $57.5 \%$. The image similarity search relies on representative images from the video shots. Based on an image corpus of more than 7 million images, we achieved a very fast response time of less than 2 seconds.

Video OCR. For the task of text retrieval, 46 query terms according to previously observed search query preferences of DRA users have been evaluated. Based on these 46 search queries, like "Abschaffung der Todesstrafe", "Kinder- und Jugendspartakiade", "Öffnungszeiten", "Protestbewegung", "Rauchen verboten", "Warschauer Vertrag", "Planerfüllung", "Nationale Front", "Gleichberechtigung", "Mikroelektronik" or "Staatshaushalt" a very satisfying retrieval performance of 92.9\% mean AP has been obtained. As expected, the results for overlaid text are significantly better than for text within the scene.

\subsection{Archivist's Perspective}

In this section, the presented content-based video retrieval system is evaluated from an archivist's perspective with a focus on usability and usefulness for archivists and DRA users. Since users of the archive are often looking for everyday scenes in the former GDR, concepts such as "pedestrian", "supermarket", "kitchen", "camping site", "allotment" or "production hall", in conjunction with the high search quality for most of these concepts, are a valuable contribution to help researchers finding appropriate scenes. Concepts with an AP score of more than approximately $50 \%$ turned out to be practically very useful. $66 \%$ of the concepts achieved an AP score of more than $50 \%$.

Searching manually for persons in videos is a quite time consuming task, particularly for less known members of the Politbüro and Ministers of the GDR. Thus, the high quality of the provided automatic person indexing algorithms is a great benefit for archivists as well as for users of the archive.

The implemented similarity search system tremendously extends the accessibility to the data in a flexible and precise way. It provides complementary search queries that are often hard to verbalize. In addition, it allows an incremental search. Previous results may serve as a source of inspiration for new similarity search queries for refining search intentions.

Another useful search option is offered by video OCR. OCR search results are very helpful since overlaid text is often closely related to the video content. The system recognizes the majority of slogans, locations and other terms precisely.

Altogether, the fine-grained automatic annotation is a very valuable supplement to human-generated meta-data. Due to the variability of the content-based video retrieval system, different user needs are taken into account. The combination of different search modalities allows to answer a wide range of user queries leading to more precise results in significantly less time. 


\section{Conclusion}

In this paper, we have presented a content-based video retrieval system for searching in historical collections of GDR television recordings. Novel algorithms for visual concept classification, similarity search, person recognition and video OCR have been developed to complement human annotations and to support users in finding relevant video shots. Experimental results on about 2,500 hours of GDR television recordings have indicated the excellent video retrieval quality in terms of AP as well as in terms of usability and usefulness from an archivist's perspective.

There are several areas for future work. First, the concept-based approach requires manually annotated training images for learning the concept models. New concepts have to be developed on demand. Therefore, it is important to reduce the effort for training data acquisition. Second, the audio modality can be used to improve the detection performance of several audio related concepts. Finally, a similarity search query can be very subjective depending on a specific user in given situation. New strategies have to be developed to predict the user's intention.

\section{Acknowledgements}

This work is financially supported by the German Research Foundation (DFGProgramm "Förderung herausragender Forschungsbibliotheken", "Bild- und Szenenrecherche in historischen Beständen des DDR-Fernsehens im Deutschen Rundfunkarchiv durch automatische inhaltsbasierte Videoanalyse"; CR 456/1-1, EW 134/1-1, FR 791/12-1).

\section{References}

1. Ahonen, T., Hadid, A., Pietikainen, M.: Face Recognition with Local Binary Patterns. In: Proc. IEEE European Conf. on Computer Vision. pp. 469-481 (2004)

2. Albertson, D., Ju, B.: Design criteria for video digital libraries: Categories of important features emerging from users' responses. Online Information Review 39(2), 214-228 (2015)

3. Belhumeur, P.N., Kriegman, D.J.: Eigenfaces vs. Fisherfaces: Recognition Using Class Specific Linear Projection. IEEE Transactions on Pattern Analysis and Machine Intelligence 19(7), 711-720 (1997)

4. Breuel, T.M., Ul-Hasan, A., Al-Azawi, M.A., Shafait, F.: High-Performance OCR for Printed English and Fraktur Using LSTM Networks. In: Proc. Int. Conf. on Document Analysis and Recognition. pp. 683-687 (2013)

5. Chatfield, K., Simonyan, K., Vedaldi, A., Zisserman, A.: Return of the Devil in the Details: Delving Deep into Convolutional Nets. In: Proceedings of the British Machine Vision Conference. pp. 1-11 (2014)

6. Christel, M., Kanade, T., Mauldin, M., Reddy, R., Sirbu, M., Stevens, S.M., Wactlar, H.D.: Informedia digital video library. Communications of the ACM 38(4), $57-58(1995)$ 
7. Chua, T.S., Tang, J., Hong, R., Li, H., Luo, Z., Zheng, Y.: NUS-WIDE: A RealWorld Web Image Database from National University of Singapore. In: Proc. ACM Int. Conf. on Image and Video Retrieval. pp. 48:1-48:9 (2009)

8. Deng, J., Dong, W., Socher, R. , Li, L.-J. , Li, K., Fei-Fei, L.: ImageNet: A LargeScale Hierarchical Image Database. In: IEEE Conference on Computer Vision and Pattern Recognition (CVPR '09). pp. 2-9 (2009)

9. Ewerth, R., Freisleben, B.: Video Cut Detection without Thresholds. In: Proceedings of the 11th International Workshop on Signals, Systems and Image Processing (IWSSIP '04). pp. 227-230. Poznan, Poland (2004)

10. Gong, Y., Jia, Y., Leung, T., Toshev, A., Ioffe, S.: Deep Convolutional Ranking for Multilabel Image Annotation. arXiv preprint arXiv:1312.4894 (2013)

11. Graves, A., Mohamed, A., Hinton, G.: Speech Recognition with Deep Recurrent Neural Networks. In: IEEE International Conference on Acoustics, Speech and Signal Processing (ICASSP 2013). pp. 6645-6649 (2013)

12. Jia, Y., Shelhamer, E., Donahue, J., Karayev, S., Long, J., Girshick, R., Guadarrama, S., Darrell, T.: Caffe: Convolutional Architecture for Fast Feature Embedding. In: Proc. ACM Int. Conf. on Multimedia. pp. 675-678 (2014)

13. Krizhevsky, A., Hinton, G.: Using Very Deep Autoencoders for Content-Based Image Retrieval. In: Proc. Europ. Symp. on Artificial Neural Networks. pp. 1-7 (2011)

14. Krizhevsky, A., Sutskever, I., Hinton, G.E.: ImageNet Classification with Deep Convolutional Neural Networks. Advances In Neural Information Processing Systems pp. 1-9 (2012)

15. Lin, K., Yang, H.f., Hsiao, J.h., Chen, C.s.: Deep Learning of Binary Hash Codes for Fast Image Retrieval. In: Proceedings of the IEEE Conference on Computer Vision and Pattern Recognition (CVPR). pp. 27-35 (2015)

16. Lowe, D.G.: Object Recognition from Local Scale-Invariant Features. In: Proc. 7th IEEE Int. Conf. on Computer Vision. vol. 2, pp. 1150-1157 (1999)

17. Marchionini, G., Geisler, G.: The open video digital library. D-Lib Magazine 8(12), 1082-9873 (2002)

18. Matas, J., Chum, O., Urban, M., Pajdla, T.: Robust wide-baseline stereo from maximally stable extremal regions. Image and Vision Computing 22(10), 761-767 (2004)

19. Mühling, M.: Visual Concept Detection in Images and Videos. Ph.D. thesis, University of Marburg (2014)

20. Mühling, M., Ewerth, R., Zhou, J., Freisleben, B.: Multimodal Video Concept Detection via Bag of Auditory Words and Multiple Kernel Learning. In: Proceedings of the 18th International Conference on Advances in Multimedia Modeling (MMM '12). pp. 40-50. Springer (2012)

21. Salakhutdinov, R., Hinton, G.: Semantic Hashing. International Journal of Approximate Reasoning 50(7), 969-978 (2009)

22. Szegedy, C., Liu, W., Jia, Y., Sermanet, P., Reed, S., Anguelov, D., Erhan, D., Vanhoucke, V., Rabinovich, A.: Going Deeper with Convolutions. In: The IEEE Conference on Computer Vision and Pattern Recognition (CVPR). pp. 1-9 (2015)

23. Taigman, Y., Yang, M., Ranzato, M., Wolf, L.: DeepFace: Closing the Gap to Human-Level Performance in Face Verification. In: Proceedings of the IEEE Conference on Computer Vision and Pattern Recognition (CVPR). pp. 1-8 (2014)

24. Viola, P., Jones, M.: Rapid Object Detection Using a Boosted Cascade of Simple Features. In: Proceedings of the 2001 IEEE Computer Society Conference on Computer Vision and Pattern Recognition. vol. 1, pp. 511-518 (2001) 
25. Wan, J., Wang, D., Hoi, S.C.H., Wu, P.: Deep Learning for Content-Based Image Retrieval: A Comprehensive Study. In: Proceedings of the ACM International Conference on Multimedia (MM). pp. 157-166 (2014)

26. Yianilos, P.N.: Data Structures and Algorithms for Nearest Neighbor Search in General Metric Spaces. In: Annual ACM-SIAM Symposium on Discrete Algorithms. pp. 311-321 (1993)

27. Zhou, B., Lapedriza, A., Xiao, J., Torralba, A., Oliva, A.: Learning Deep Features for Scene Recognition Using Places Database. Advances in Neural Information Processing Systems 27 pp. 487-495 (2014) 